\title{
Primary Extraosseous Ewing Sarcoma of the Upper Limb: Report of a Rare Case
}

\author{
Ahmad R Mafi (iD ${ }^{1,{ }^{*}, \text { Hasan Barati }}{ }^{2,{ }^{* *}}$ and Keyvan Ramezani ${ }^{2}$ \\ ${ }^{1}$ Department of Radiation Oncology, Imam Hossein Hospital, Shahid Beheshti University of Medical Sciences, Tehran, Iran \\ ${ }^{2}$ Department of Orthopedic Surgery, Imam Hossein Hospital, Shahid Beheshti University of Medical Sciences, Tehran, Iran \\ "Corresponding author: Department of Radiation Oncology, Imam Hossein Hospital, Shahid Beheshti University of Medical Sciences, Tehran, Iran. Email: \\ ahmadrmafi@yahoo.com \\ "* Corresponding author: Department of Orthopedic Surgery, Imam Hossein Hospital, Shahid Beheshti University of Medical Sciences, Tehran, Iran. Email: \\ dr.barati363@gmail.com
}

Received 2021 January 30; Revised 2021 May 09; Accepted 2021 May 30.

\begin{abstract}
Introduction: Extraosseous Ewing sarcomas (EESs) are rare tumors that originate from soft tissues. Upper extremity EESs account for about $3 \%$ of all cases. Here we reported a case of ESS of the upper limb whose management became complicated due to the COVID-19 pandemic.

Case Presentation: A 27-year-old female with EES of the right deltoid region presented after 3 months delay when the tumor had reached a huge size. Neoadjuvant therapy was initiated for her with acceptable results, however, her surgical treatment was postponed 3 times due to the cancellation of elective operations in the hospital as well as her involvement with COVID-19 infection. She developed multiple pulmonary metastases shortly after the surgery and passed away within a fortnight due to respiratory complications.

Conclusions: Although not "emergent" by definition, surgical treatment of patients with cancer, especially those who suffer from malignancies with high metastatic potential such as Ewing sarcoma (including EES), should not be considered as "elective" since the disease may progress in a short time and become incurable.
\end{abstract}

Keywords: Extraosseous, Ewing Sarcoma, COVID 19

\section{Introduction}

Ewing sarcoma (ES) is a highly aggressive round cell tumor that lacks cellular or structural differentiation. ES is the second most common malignant bone tumor in children and young adults that usually shows a high rate of local recurrence and distant metastasis (1).

Extraosseous Ewing sarcomas (EESs) are rare tumors that originate from soft tissues and upper extremity EESs account for about $3 \%$ of all cases (2).

We reported a 27-year-old female with EES of the right deltoid region that unfortunately, management of her potentially curable disease became complicated due to the COVID-19 pandemic.

\section{Case Presentation}

A 27-year-old female presented with progressive swelling and pain in the right deltoid for more than 4 months. As her signs and symptoms had begun following minor trauma, and besides, due to the COVID-19 outbreak, she had more than 3 months delay in seeking medical advice.

Her physical examination revealed a $20 \times 15 \mathrm{~cm}$ firm and tender swelling over the left deltoid region that has caused moderate pain and severe limitation on range of the motion.

Other parts of the physical examination were unremarkable and all her laboratory tests were within normal limits.

Magnetic resonance imaging (MRI) of the affected arm showed a well-defined hyperintense hemorrhagic mass lesion in the lateral part of the upper muscle of the arm, highly suspicious of a malignant legion with the normal fat plane and adjacent bone (Figure $1 \mathrm{~A}$ and $\mathrm{B}$ ).

A biopsy was taken, and the final diagnosis of Ewing sarcoma was made (Figure 2A - C). Metastasis workup showed no metastatic lesion. Neoadjuvant chemotherapy with VAC-IE regimen (vincristine, doxorubicin, and cyclophosphamide, alternating with ifosfamide, and etoposide every 3 weeks) was started. The lesion responded well to the treatment with more than $50 \%$ size reduction after 

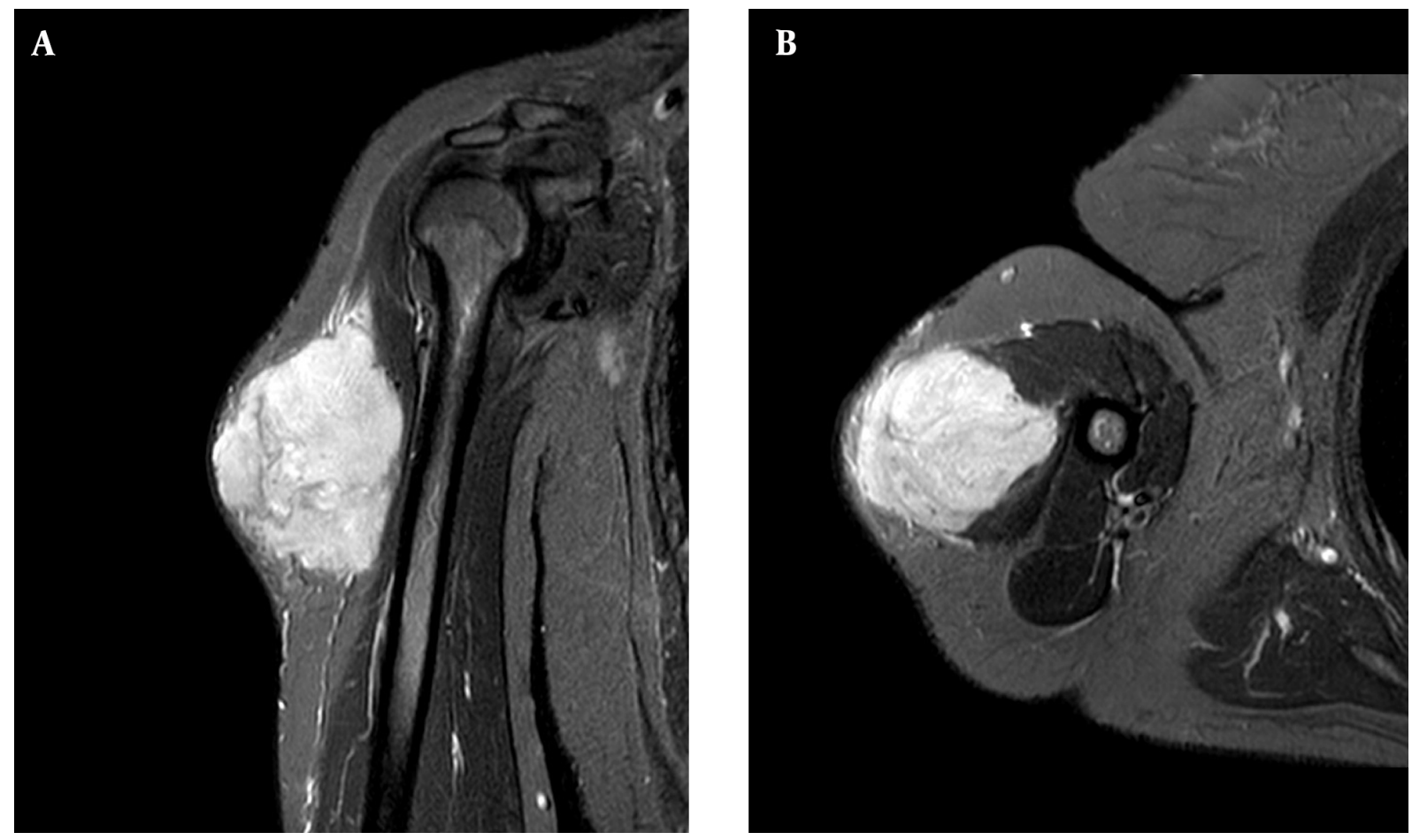

Figure 1. A and B, well-defined hyperintense hemorrhagic mass lesion in the lateral part of the upper muscle of the arm, highly suspicious of a malignant lesion. Adjacent bone appears intact and the fat plane is normal and the bone marrow shows normal signal intensity.

\section{2 cycles of chemotherapy.}

During the third cycle, the lesion began to pain and grow. We discussed this case in the multidisciplinary meeting and finally, we decided that she should undergo surgery.

Since all the elective surgeries at that time were canceled due to the COVID-19 pandemic, chemotherapy was resumed with a different regimen (irinotecan and temozolomide), while she was still non-metastatic based on restaging scans.

Following the second course of the second-line chemotherapy, she developed neutropenic fever and was hospitalized for 10 days.

Two weeks after the discharge, in the scheduled surgery time, she was infected with COVID-19 and was admitted to the intensive care unit (ICU) due to the severe symptoms. She was discharged after 22 days and stayed at home for more than 20 days because of poor performance status. Meanwhile, the lesion began to grow again and it measured $40 \times 30 \mathrm{~cm}$ when she was admitted for surgery.

Her surgical treatment was canceled once more due to a lack of ICU beds.

Finally, about 5 months after the initial visit, right shoulder disarticulation surgery was performed for her (Figure 3).
After the surgery, she stayed in the ICU for 10 days due to respiratory distress. About 3 weeks following the surgery she was well enough to continue the chemotherapy. The chemotherapy was resumed with the same regimen as the second-line treatment. During the second course, she developed respiratory distress and the chest computed tomography (CT) scan revealed multiple metastatic lesions in both lungs as well as the residual ground-glass opacities of the COVID-19 infection (Figure 4). She was admitted to the ICU and passed away 12 days afterward.

\section{Discussion}

In this paper, we reported a rare but potentially curable case of EES whose management was complicated due to the COVID-19 pandemic.

Ewing sarcoma is a poorly differentiated, highly malignant and round cell tumor which lacks cellular or structural differentiation (3). Although, it is primarily a bone tumor, a large-scale analysis of the surveillance, epidemiology and end results (SEER) database demonstrated that up to $31 \%$ of cases might arise from extra-skeletal tissues (4).

Extraosseous Ewing sarcomas are rare tumors that originate from soft tissues and most often involve paraver- 

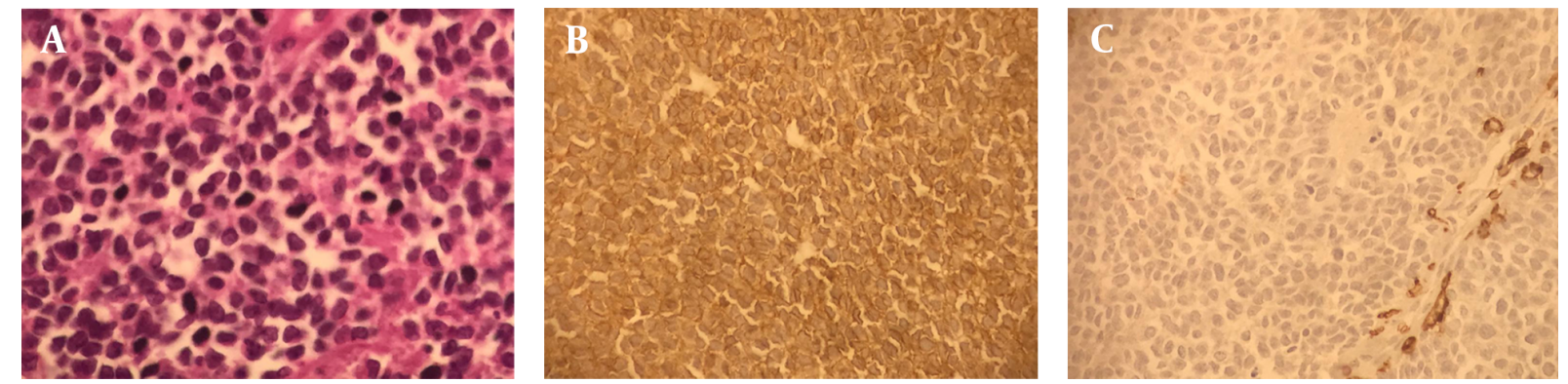

Figure 2. A, sheets of small round cells with scant cytoplasm and indistinct cell membranes having minimal amount of stroma (microscopic image at $\mathrm{x} 40$ ); B, tumor cells showing membrane positively for CD 99 (microscopic image at x40); C, tumor cells are negative for ICA, cytokeratin, chromgranin and synaptophysin

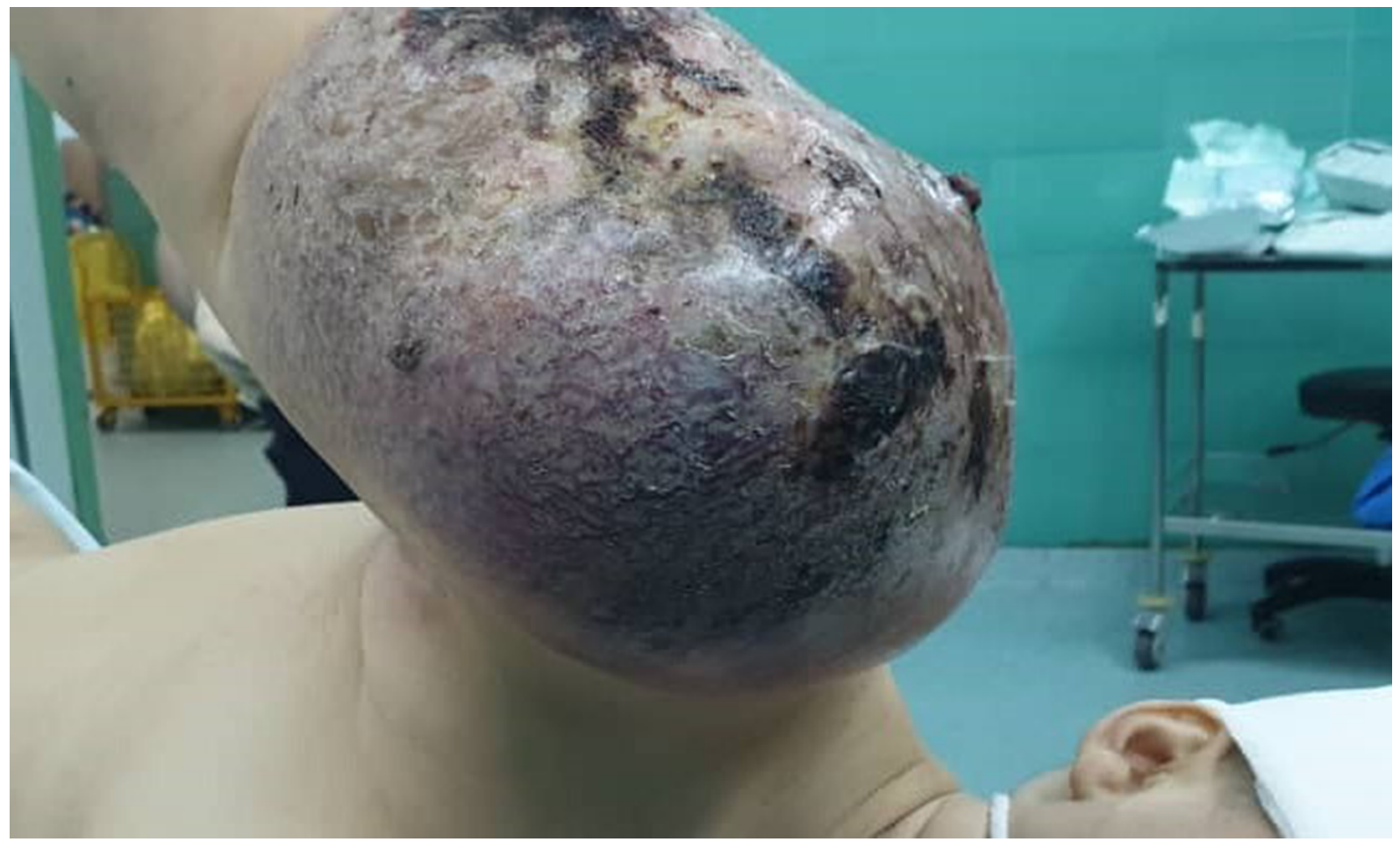

Figure 3. Right arm's mass in the operating room

tebral spaces, lower extremities, head and neck, and pelvis and upper extremity EESs account for about $3 \%$ of all cases $(2-4)$.

Signs and symptoms of EES depend on the site of origin and is commonly due to the mass effects, including pain and swelling (4).

The presentation of EES is somewhat different from that of osseous ES. While ES generally presents in the second decade of life with a slight female predilection, EES has a bimodal age distribution and is commonly found in patients younger than 5 years and older than 35 years, with no gender preference $(4,5)$.

Radiographic findings of EES include increased soft tissue density without any bony involvement with calcification in about $25 \%$ of the cases. MRI is useful in evaluating the extent of the tumor and its relation with the neurovascular bundles. On MRI, EES is often of low to intermediate signal intensity on T1-weighted images and of high signal intensity on T2-weighted images and shows heterogeneous contrast enhancement (1-3).

One important characteristic of EESs is that although they can cause cortical erosion and/or a periosteal reac- 


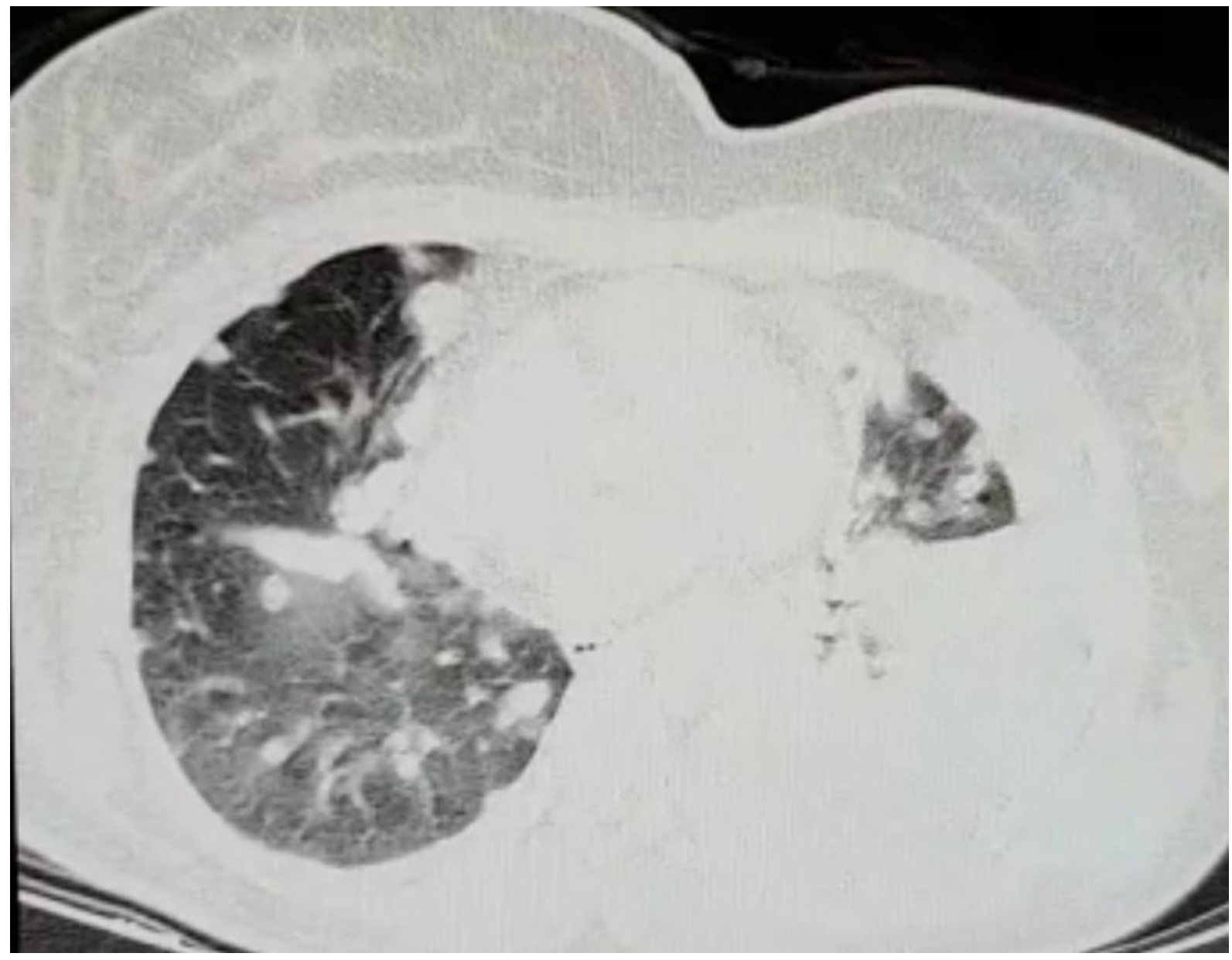

Figure 4. Chest CT scan showing multiple lung metastases and ground glass opacities

tion, actual osseous involvement is rare, even when the mass is located near the bone (3).

The recommended treatment for any member of the Ewing tumor family is local treatment with surgery (or radiotherapy for inoperable cases) plus systemic therapy. Based on the most international clinical guidelines, neoadjuvant chemotherapy followed by aggressive surgical excision with or without radiation therapy is the preferred treatment method for the majority of the patients $(1,3)$.

By standard treatment, ES and EES have similar prognosis in general, with 5-year overall survival (OS) of 65 to $75 \%$ for localized disease, and less than $30 \%$ for metastatic disease (6-8). However, some studies have reported better event-free and overall survival rates for EES (9).

Timely commencement of systemic therapy along with aggressive local therapy (mainly surgery, and in some cases radiotherapy) are of paramount importance in achieving survival benefits. One study on 27 patients with
EES reported that patients who had more than 90\% tumor necrosis (less than 10\% viable tumor) had a 100\% 5-year OS. Besides, patients who received induction chemotherapy and underwent wide surgical resection with negative margins had also a 100 \% 5-year OS (10).

According to the majority of international clinical guidelines, we started our patient's treatment with neoadjuvant chemotherapy with the hope of controlling the systemic disease, as well as increasing the chance of limb preservation and achieving negative surgical margins. Unfortunately, the COVID-19 pandemic took her medical management out of our control to a great extent.

Our patient initially had a 3-month delay in seeking medical advice due to COVID-19 fear. Based on the recently published data, every 4 weeks delay in diagnosis and treatment of patients with different types of cancers, can result in a 6 to 13\% higher risk of death (11-13).

Furthermore, reports demonstrated that patients with 
cancer not only are at greater risk of contracting COVID-19 but also are more susceptible to develop severe infection and as a result, have a significantly higher mortality rate from COVID-19 (14).

This high risk of mortality could be due to immunosuppression, increased co-existing medical conditions, and pulmonary compromise in cases of involvement of lungs with malignancy (14).

In addition, experts suggest that severe COVID-19 may create a microenvironment favorable to cancer recurrence by immune-mediated tumor reawakening (15).

COVID-19 can activate several factors that have been previously shown to have a role in tumorigenesis and metastatic relapse. Recent studies have revealed that COVID-19 can target common cancer pathways including those involved in cell cycle progression, metabolism, and epigenetics (15). This finding will further complicate the interaction between COVID-19 and malignant diseases.

It can be assumed that several factors including delayed diagnosis and treatment commencement, higher susceptibility of contracting COVID-19, treatment interruptions due to shortage of hospital bed and healthcare personnel, more severe respiratory infection due to malignancy, the possible role of COVID-19 in tumor reawakening, and finally, aggressive behavior of her cancer, altogether prevented us from achieving our desired treatment result and led to the patient death.

\subsection{Conclusions}

Extraosseous Ewing sarcoma is a rare but curable disease. COVID-19 pandemic has affected all aspects of medical care including the management of patients with cancer. Although not "emergent" by definition, surgical treatment of patients with cancer, especially those who suffer from malignancies with high metastatic potential such as Ewing sarcoma (including EES) should not be considered as "elective", as the disease may progress in a short time and become incurable.

\section{Footnotes}

Authors' Contribution: Study concept and design, A.R.M, H.B and K.R; Manuscript draft, A.R.M, H.B and K.R; Critical revision of the manuscript for important intellectual content, A.R.M

Conflict of Interests: There is no conflict of interest.

Ethical Approval: As you have mentioned in the instructions, case reports are not obliged to show Ethical Approval Code.

Funding/Support: There was no funding/support. Informed Consent: Written informed consent was obtain from the patient.

\section{References}

1. Bansal K, Prasad A, Shahi P, Sehgal A, Kamal S. Extraosseus Ewing's sarcoma of the forearm. Cureus. 2020;12(7). e9051. doi: 10.7759/cureus.9051. [PubMed: 32782870]. [PubMed Central: PMC7410401].

2. Murphey MD, Senchak LT, Mambalam PK, Logie CI, Klassen-Fischer MK, Kransdorf MJ. From the radiologic pathology archives: Ewing sarcoma family of tumors: Radiologic-pathologic correlation. Radiographics. 2013;33(3):803-31. doi: 10.1148/rg.333135005. [PubMed: 23674776].

3. Galyfos G, Karantzikos GA, Kavouras N, Sianou A, Palogos K, Filis K. Extraosseous Ewing sarcoma: Diagnosis, prognosis and optimal management. Indian J Surg. 2016;78(1):49-53. doi: 10.1007/s12262-015-13990. [PubMed: 27186040]. [PubMed Central: PMC4848231].

4. McArdle DJT, Nott L, Harle R, McArdle JP. Extraosseous Ewing sarcoma arising in a chronically lymphedematous limb. J Vasc Surg Cases Innov Tech. 2018;4(3):210-5. doi: 10.1016/j.jvscit.2018.04.005. [PubMed: 30148241]. [PubMed Central: PMC6105752].

5. Yao L, Tang J, Lu L, Sayfoo M, Zhu X. Primary extraskeletal Ewing sarcoma of the sinonasal tract: A rare case report and review of the literature. Int J Clin Exp Med. 2018;11(5):256-62.

6. Lee JA, Kim DH, Lim JS, Koh JS, Kim MS, Kong CB, et al. Soft-tissue Ewing sarcoma in a low-incidence population: comparison to skeletal Ewing sarcoma for clinical characteristics and treatment outcome. Jpn JClin Oncol. 2010;40(11):1060-7. doi:10.1093/jjco/hyq080. [PubMed: 20513751].

7. Gaspar N, Hawkins DS, Dirksen U, Lewis IJ, Ferrari S, Le Deley $\mathrm{MC}$, et al. Ewing sarcoma: Current management and future approaches through collaboration.JClin Oncol. 2015;33(27):3036-46. doi: 10.1200/JCO.2014.59.5256. [PubMed: 26304893].

8. El Weshi A, Allam A, Ajarim D, Al Dayel F, Pant R, Bazarbashi S, et al. Extraskeletal Ewing's sarcoma family of tumours in adults: Analysis of 57 patients from a single institution. Clin Oncol. 2010;22(5):374-81. doi: 10.1016/j.clon.2010.02.010. [PubMed: 20466282].

9. Biswas B, Shukla NK, Deo SV, Agarwala S, Sharma DN, Vishnubhatla S, et al. Evaluation of outcome and prognostic factors in extraosseous Ewing sarcoma. Pediatr Blood Cancer. 2014;61(11):1925-31. doi: 10.1002/pbc.25095. [PubMed: 25132242].

10. Tural D, Molinas Mandel N, Dervisoglu S, Oner Dincbas F, Koca S, Colpan Oksuz D, et al. Extraskeletal Ewing's sarcoma family of tumors in adults: Prognostic factors and clinical outcome. Jpn J Clin Oncol. 2012;42(5):420-6. doi:10.1093/jjco/hys027. [PubMed: 22416252].

11. Maringe C, Spicer J, Morris M, Purushotham A, Nolte E, Sullivan $\mathrm{R}$, et al. The impact of the COVID-19 pandemic on cancer deaths due to delays in diagnosis in England, UK: A national, population-based, modelling study. Lancet Oncol. 2020;21(8):1023-34. doi: 10.1016/S1470-2045(20)30388-0. [PubMed: 32702310]. [PubMed Central: PMC7417808].

12. Maddumarachchi PS, Somathilake M, Gunasekera S. Impact of treatment delays in Ewings sarcoma in a low resourse setting. Ann Oncol. 2019;30(Suppl 6):vi90-vi117. doi: 10.1093/annonc/mdz338.002.

13. Hanna TP, King WD, Thibodeau S, Jalink M, Paulin GA, Harvey-Jones E, et al. Mortality due to cancer treatment delay: Systematic review and meta-analysis. BMJ. 2020;371:m4087. doi: 10.1136/bmj.m4087. [PubMed: 33148535]. [PubMed Central: PMC7610021].

14. Addeo A, Friedlaender A. Cancer and COVID-19: Unmasking their ties. Cancer Treat Rev. 2020;88:102041. doi: 10.1016/j.ctrv.2020.102041. [PubMed: 32516704]. [PubMed Central: PMC7831797].

15. Francescangeli F, De Angelis ML, Baiocchi M, Rossi R, Biffoni M, Zeuner A. COVID-19-induced modifications in the tumor microenvironment: Do they affect cancer reawakening and metastatic relapse? Front Oncol. 2020;10:592891. doi: 10.3389/fonc.2020.592891. [PubMed: 33194755]. [PubMed Central: PMC7649335]. 